\title{
ASSOCIAÇÃO ENTRE AS CARACTERÍSTICAS SOCIODEMOGRÁFICAS E A CAPACIDADE FUNCIONAL DE IDOSOS LONGEVOS DA COMUNIDADE
}

\author{
Maria Helena Lenardt ${ }^{1}$, Nathalia Hammerschmidt Kolb Carneiro ${ }^{2}$
}

\begin{abstract}
RESUMO: Trata-se de estudo quantitativo de corte transversal, cujo objetivo foi relacionar a capacidade funcional entre variáveis sociodemográficas dos idosos longevos usuários da Atenção Básica de Saúde. A amostra foi constituída por 65 idosos de $\geq 80$ anos no período amostral de três meses e mediante critérios de inclusão e exclusão. A coleta de dados ocorreu em Unidade Básica de Saúde e domicílios, em 2011, por meio da aplicação de questionário sociodemográfico e escala de Medida de Independência Funcional. Os resultados foram considerados estatisticamente significativos quando $\mathrm{p}<0,05$. As variáveis sociodemográficas significativas para capacidade funcional foram: idade ( $\mathrm{p}=0,044)$, local de nascimento $(\mathrm{p}=0,029)$, com quem mora ( $\mathrm{p}=0,050)$, renda $(\mathrm{p}=0,048)$ e trabalho $(\mathrm{p}=0,050)$. Os resultados reforçam a importância de se considerar, durante as ações de cuidados, a interferência de características sociais e demográficas na capacidade funcional dos idosos longevos; elementar para a efetividade e eficácia do cuidado.
\end{abstract}

DESCRITORES: Idoso de 80 anos ou mais; Longevidade; Centros de saúde; Enfermagem geriátrica.

\section{ASSOCIATION BETWEEN SOCIO-DEMOGRAPHIC CHARACTERISTICS AND THE FUNCTIONAL ABILITY OF VERY ELDERLY PERSONS IN THE COMMUNITY}

ABSTRACT: This is a quantitative, cross-sectional study, aiming to relate functional ability with the socio-demographic variables of the very elderly service users of Primary Health Care. The sample was made up of 65 older adults $\geq 80$ years of age in the sampling period of three months, and following inclusion and exclusion criteria. Data was collected in the Primary Health Care center and in the older adults' homes, in 2011, through the administration of a socio-demographic questionnaire and the Functional Independence Measure scale. The results were considered statistically significant when $\mathrm{p}<0.05$. The socio-demographic variables which were significant for functional capacity were: age $(\mathrm{p}=0.044)$, place of $\operatorname{birth}(\mathrm{p}=0.029)$, with whom the person lives $(\mathrm{p}=0.050)$, income $(\mathrm{p}=0.048)$ and work $(\mathrm{p}=0.050)$. The results support the importance of taking into account, during care actions, how social and demographic characteristics influence the functional capacity of very elderly persons; this is elementary to the effectiveness and efficacy of the care.

DESCRIPTORS: Elderly aged 80 or over; Longevity; Health centers; Geriatric nursing.

\section{ASOCIACIÓN ENTRE LAS CARACTERÍSTICAS SOCIODEMOGRÁFICAS Y LA CAPACIDAD FUNCIONAL DE ANCIANOS LONGEVOS DE LA COMUNIDAD}

RESUMEN: Este es un estudio cuantitativo de corte transversal, cuyo objetivo fue relacionar la capacidad funcional entre variables sociodemográficas de los ancianos longevos usuarios de la Atención Básica de Salud. La muestra fue constituida por 65 ancianos de $\geq 80$ años en el periodo de tres meses y por medio de criterios de inclusión y exclusión. Los datos fueron recogidos en Unidad Básica de Salud y domicilios, en 2011, por medio de la aplicación de cuestionario sociodemográfico y escala de Medida de Independencia Funcional. Los resultados fueron considerados estadisticamente significativos cuando $p<0,05$. Las variables sociodemográficas significativas para capacidad funcional fueron: edad $(\mathrm{p}=0,044)$, local de nacimiento $(\mathrm{p}=0,029)$, con quien vive $(\mathrm{p}=0,050)$, renta $(\mathrm{p}=0,048)$ y trabajo $(\mathrm{p}=0,050)$. Los resultados resaltan la importancia de considerarse, durante las acciones de cuidados, la interferencia de características sociales y demográficas en la capacidad funcional de los ancianos longevos; elementar para la efectividad y eficacia del cuidado.

DESCRIPTORES: Anciano de 80 años o más; Longevidad; Centros de salud; Enfermería geriátrica.

${ }^{1}$ Enfermeira. Doutora em Filosofia da Enfermagem. Professora do Programa de Pós-Graduação em Enfermagem da Universidade Federal do Paraná - UFPR. Líder do Grupo Multiprofissional de Pesquisa sobre Idosos - GMPI.

${ }^{2}$ Enfermeira. Mestranda em Enfermagem pelo Programa de Pós-Graduação em Enfermagem da UFPR. Bolsista REUNI. Membro do GMPI. 


\section{INTRODUÇÃO}

Observam-se na literatura diversos termos para caracterizar o idoso que possui mais de 80 anos, entre eles: idoso muito idoso, idoso mais idoso, idoso mais velho, idoso de 80 anos ou mais, octagenário e idoso longevo ${ }^{(1-2)}$. Essa diferenciação dos idosos longevos dos idosos com mais de 60 anos ocorreu pelo aumento crescente dessa parcela da população. Além disso, os idosos com 80 anos ou mais apresentam características morfofisiológicas, psicológicas e socioeconômicas diferenciadas de outros indivíduos, inclusive dos idosos 'mais jovens ${ }^{\text {(3) }}$.

Apesar de se observar aumento da população desse segmento etário, verifica-se que o número de estudos com enfoque nos idosos com 80 anos ou mais mostra-se bastante reduzido. Tal constatação significa que, no Brasil, ainda é pouca a preocupação com as questões pertinentes ao idoso longevo ${ }^{(4)}$.

Os estudos publicados sobre a independência têm projetado um alto crescimento da população idosa funcionalmente incapacitada. Sabe-se que com o passar dos anos de vida há um aumento significativo de dependência, isto é, os grupos etários mais velhos são proporcionalmente mais dependentes que os mais jovens ${ }^{(5)}$. Dessa maneira, além das doenças crônicas aumentarem a prevalência das incapacidades, o próprio envelhecimento diminui a capacidade funcional de cada sistema do organismo; o que se denomina envelhecimento funcional.

Entende-se por capacidade funcional "a condição que o indivíduo possui de viver de maneira autônoma e de se relacionar em seu meio"(6:323). A perda da capacidade funcional relaciona-se, principalmente, ao maior risco de institucionalização e quedas ${ }^{(7)}$, sendo considerado fator de risco independente para a mortalidade em idosos longevos ${ }^{(8)}$.

Alguns estudos internacionais avaliam a relação da capacidade funcional dos idosos com a idade dos participantes. Entre eles, está uma investigação ${ }^{(9)}$ realizada com 203 idosos longevos suecos, a qual objetivou identificar os problemas de saúde físicos e funcionais dos idosos e sua relação entre as atividades de vida diária. Como resultado, o estudo demonstrou que $36 \%$ desses idosos longevos possuíam dificuldade na realização de pelo menos uma das atividades de vida diária ${ }^{(9)}$. Outro estu$\mathrm{do}^{(10)}$, realizado com 8.805 idosos chineses na faixa etária entre 80 e 105 anos, observou que os idosos oriundos da zona rural possuem níveis de atividade de vida diária maiores do que aqueles residentes na zona urbana, podendo ser considerados idosos mais ativos.

Além da faixa etária e local de nascimento, acredita-se que outros dados sociodemográficos também interfiram na capacidade funcional dos idosos. Um estudo nacional, realizado com 964 indivíduos de 60 anos ou mais residentes em São Paulo, observou que a dependência dos idosos estava relacionada a algumas características específicas, como: baixa escolaridade, aposentadoria, não ter moradia própria e possuir idade superior a $65 \operatorname{anos}^{(11)}$.

Desse modo, a relevância deste estudo consiste em conhecer a capacidade funcional dos idosos longevos usuários da Atenção Básica de Saúde e suas relações às características sociodemográficas, haja vista que a obtenção desses dados e suas análises fornecem subsídios, principalmente locais, para o desenvolvimento de novas pesquisas. Outrossim, permite aos profissionais de enfermagem atuarem na promoção à saúde com foco na funcionalidade do idoso, bem como na elaboração de um plano de cuidado individualizado, que contemple as reais especificidades deles, para além das características somente fisiológicas.

Diante destas considerações, o objetivo deste estudo foi relacionar a capacidade funcional entre variáveis sociodemográficas dos idosos longevos usuários da Atenção Básica de Saúde.

\section{MÉTODO}

Trata-se de estudo quantitativo de corte transversal realizado com idosos longevos de 80 anos ou mais, de ambos os sexos, usuários de uma Unidade Básica de Saúde (UBS) de uma capital brasileira, no período amostral de três meses. A população de idosos de 80 anos ou mais cadastrados em programas oferecidos por essa UBS era constituída de 107 idosos longevos. Desse modo, todos os idosos longevos foram considerados com possibilidade de participantes do estudo.

Para a seleção dos idosos, utilizaram-se os seguintes critérios de inclusão: possuir idade igual ou superior a 80 anos; estar cadastrado na unidade de saúde selecionada para o estudo; ter capacidade para responder os instrumentos utilizados (podendo o cuidador e/ou familiar complementar a resposta, exceto no questionário que avalia a função cognitiva do idoso - Mini Exame do Estado Mental). E 
os critérios de exclusão: obter pontuação inferior ao ponto de corte no Mini Exame do Estado Mental (MEEM); apresentar dificuldade de comunicação que impossibilite a entrevista; expressar, voluntariamente e a qualquer momento, o desejo de interromper sua participação no estudo.

Para elegibilidade do cuidador e/ou familiar do idoso longevo foram elaborados os seguintes critérios de inclusão: constatar dificuldade de comunicação e alteração cognitiva do idoso longevo; ser o cuidador e/ou familiar e residir com o idoso longevo; possuir idade igual ou superior a 18 anos. Critérios de exclusão do cuidador: apresentar dificuldade de comunicação que impossibilite a entrevista; expressar, voluntariamente e a qualquer momento, o desejo de interromper sua participação no estudo.

O Mini Exame do Estado Mental foi utilizado para o rastreamento de alteração da função cognitiva (screening cognitivo) dos idosos. Esse exame compreende 11 itens, agrupados em sete categorias: orientação temporal, orientação espacial, registro de três palavras, atenção e cálculo, lembrança das três palavras, linguagem e capacidade construtiva visual. A pontuação é de zero a $30^{(12)}$. Neste estudo, empregou-se a versão validada que utiliza os seguintes pontos de corte: 13 para analfabetos, 18 para média e baixa escolaridade e 26 para alta escolaridade ${ }^{(13)}$.

A coleta de dados ocorreu no período de agosto a outubro de 2011, na UBS e nos domicílios, por meio dos seguintes instrumentos: questionário sociodemográfico e escala de Medida de Independência Funcional (MIF). O questionário sociodemográfico foi elaborado pelos autores e abrange as seguintes variáveis: sexo, idade, local de nascimento, cor ou raça, escolaridade, estado civil, com quem mora, renda familiar, trabalho, filhos e religião.

Para a avaliação da capacidade funcional, utilizou-se a Medida de Independência Funcional (MIF) traduzida e adaptada para o Brasil ${ }^{(14)}$. A escala verifica o desempenho da pessoa idosa para a realização de um conjunto de 18 tarefas, referentes às subescalas de autocuidado, controle esfincteriano, transferências, locomoção, comunicação e cognição social. Nas tarefas da MIF cada item pode ser classificado em uma escala de graus de dependência de 7 níveis, sendo o valor 1 correspondente à dependência total e o valor 7 correspondente à normalidade na realização de tarefas de forma independente. Deste modo, o valor total varia de
18 (totalmente dependentes) à 126 (completamente independentes) ${ }^{(15)}$.

A amostra final do estudo constituiu-se de 65 idosos longevos, entrevistados aleatoriamente no período de agosto a outubro de 2011, conforme os critérios de inclusão e exclusão da pesquisa. Dos 65 idosos longevos, $9(13,8 \%)$ apresentaram alteração cognitiva rastreada através do MEEM ou estavam acamados e não responsivos. Nestes casos o cuidador/familiar foi convidado a participar.

Os dados foram organizados e armazenados no software Excel $^{\circledR} 2007$. Para a análise dos resultados, utilizou-se o software EpiInfo versão 6.04 e aplicou-se estatística descritiva, por meio da distribuição da frequência absoluta e percentual, média e desvio padrão, e testes não paramétricos (Qui-quadrado e Kruskal-Wallis H) de associação de variáveis. Os resultados foram considerados estatisticamente significativos quando $\mathrm{p}<0,05$.

O estudo recebeu parecer favorável do Comitê de Ética em Pesquisa em Seres Humanos do Setor de Ciências da Saúde, sendo aprovado sob o registro CEP/SD 1080.105.11.08 e CAAE 0104.0.091.085-11. Foram respeitados os preceitos éticos de participação voluntária e consentida.

\section{RESULTADOS}

Aponta-se no quadro 1 as associações entre as características sociodemográficas dos participantes e a capacidade funcional.

Observa-se que a maioria dos idosos longevos é do sexo feminino ( $\mathrm{n}=35 ; 53,8 \%)$, com prevalência da faixa etária entre 80 e 84 anos $(n=37 ; 56,9 \%)$, oriundos da área rural $(n=41 ; 63,1 \%)$, com ensino fundamental incompleto $(\mathrm{n}=35 ; 53,9 \%)$, viúvos $(n=42 ; 64,6 \%)$, residem com familiares $(n=30$; $46,2 \%)$ e tem renda de um salário mínimo $(n=23$; 35,4\%) (Quadro 1).

Visualiza-se na quadro 1 a associação entre os valores da MIF e as variáveis sociodemográficas dos participantes e consta-se que no domínio motor somente a variável 'local de nascimento' apresentou significância estatística $(p=0,037)$. No domínio cognitivo, nenhuma das variáveis sociodemográficas foi estatisticamente significativa. Quanto à MIF total as variáveis estatisticamente significativas foram: idade $(\mathrm{p}=0,044)$; local de nascimento $(\mathrm{p}=0,029)$; com quem mora $(\mathrm{p}=0,050)$; renda $(\mathrm{p}=0,048)$; e trabalho $(\mathrm{p}=0,050)$. 
Quadro 1-Associações entre as características sociodemográficas e a capacidade funcional dos idosos longevos. Curitiba, 2011

\begin{tabular}{|c|c|c|c|c|c|c|}
\hline \multirow{2}{*}{ Características } & \multirow{2}{*}{ Classificação } & \multirow{2}{*}{$\mathbf{n}$} & \multirow{2}{*}{$\%$} & \multicolumn{3}{|c|}{$P$ valor* } \\
\hline & & & & MIFm** & MIFc** & MIFt** \\
\hline \multirow{2}{*}{ Sexo } & Feminino & 35 & 53,8 & \multirow{2}{*}{0,751} & \multirow{2}{*}{0,599} & \multirow{2}{*}{0,692} \\
\hline & Masculino & 30 & 46,2 & & & \\
\hline \multirow{3}{*}{ Idade } & 80 a 84 anos & 37 & 56,9 & \multirow{3}{*}{0,059} & \multirow{3}{*}{0,097} & \multirow{3}{*}{$0,044^{*}$} \\
\hline & 85 a 89 anos & 24 & 36,9 & & & \\
\hline & 90 anos ou mais & 04 & 6,2 & & & \\
\hline \multirow{2}{*}{ Local de nascimento } & Rural & 41 & 63,1 & \multirow{2}{*}{$0,037^{*}$} & \multirow{2}{*}{0,086} & \multirow{2}{*}{$0,029^{*}$} \\
\hline & Urbano & 24 & 36,9 & & & \\
\hline \multirow{3}{*}{ Cor ou raça } & Branca & 53 & 81,5 & \multirow{3}{*}{0,875} & \multirow{3}{*}{0,835} & \multirow{3}{*}{0,829} \\
\hline & Parda & 10 & 15,4 & & & \\
\hline & Preta & 02 & 3,1 & & & \\
\hline \multirow{6}{*}{ Escolaridade } & $1^{\circ}$ grau incompleto & 35 & 53,9 & \multirow{6}{*}{0,960} & \multirow{6}{*}{0,587} & \multirow{6}{*}{0,962} \\
\hline & Analfabeto (a) & 28 & 43,1 & & & \\
\hline & $1^{\circ}$ grau completo & - & - & & & \\
\hline & $2^{\circ}$ grau incompleto & 01 & 1,5 & & & \\
\hline & $2^{\circ}$ grau completo & 01 & 1,5 & & & \\
\hline & Ensino superior & - & - & & & \\
\hline \multirow{4}{*}{ Estado civil } & Viúvo (a) & 42 & 64,6 & & & \\
\hline & Casado (a) & 20 & 30,8 & & & \\
\hline & Separado (a) & 02 & 3,1 & 0,808 & 0,500 & 0,543 \\
\hline & Solteiro (a) & 01 & 1,5 & & & \\
\hline & Familiares & 30 & 46,2 & & & \\
\hline Com auem mora & Sozinho (a) & 19 & 29,2 & 0053 & 0210 & $0050 *$ \\
\hline Com quem mora & Cônjuge & 08 & 12,3 & 0,053 & 0,210 & $0,050^{*}$ \\
\hline & Filho (a) & 08 & 12,3 & & & \\
\hline & 1 salário mínimo & 23 & 35,4 & & & \\
\hline Dond fomilion & 2 salários mínimos & 16 & 24,6 & 0067 & 0220 & $0048 *$ \\
\hline Renda raminar & 3 salários mínimos & 16 & 24,6 & $0,06 /$ & $0,2 \angle 0$ & $0,048^{*}$ \\
\hline & $>4$ salários mínimos & 10 & 15,4 & & & \\
\hline Possui algum & Não & 62 & 95,4 & 0106 & 0077 & $0050 *$ \\
\hline trabalho & Sim & 03 & 4,6 & 0,100 & $0,0 / 1 /$ & 0,000 \\
\hline Possui filhos & Sim & 59 & 90,8 & 0.846 & 0.410 & 0.666 \\
\hline Possul ninos & Não & 06 & 9,2 & 0,840 & 0,410 & 0,060 \\
\hline & Católica & 45 & 69,2 & & & \\
\hline & Evangélica & 17 & 26,3 & & & \\
\hline Religião & Espírita & 01 & 1,5 & 0,872 & 0,787 & 0,831 \\
\hline & Luterana & 01 & 1,5 & & & \\
\hline & Não possui & 01 & 1,5 & & & \\
\hline Total & - & 65 & 100 & - & - & - \\
\hline
\end{tabular}

* Teste de Kruskal-Wallis H.

** Medida de Independência Funcional (MIF) motora, cognitiva e total. 


\section{DISCUSSÃO}

A predominância do sexo feminino, viúvas e na faixa etária de 80 a 84 anos foi semelhante a alguns resultados encontrados na literatura. Os resultados de um estudo, realizado no Estado de São Paulo com 80 idosos longevos da comunidade, apontaram que $72,5 \%$ dos longevos eram do sexo feminino e $27,5 \%$ do sexo masculino ${ }^{(16)}$. Da mesma maneira, outra investigação que entrevistou 2.924 idosas octagenárias residentes em Uberaba - Minas Gerais observou que $73,8 \%$ da sua amostra era composta por idosas viúvas ${ }^{(17)}$.

De acordo com os dados do Instituto Brasileiro de Geografia e Estatística, no ano de 2008, a expectativa de vida ao nascer, para o sexo masculino, era de 69,09 anos, ao passo que para o sexo feminino, era de 76,66 anos, havendo uma diferença de 7,06 anos entre os dois sexos ${ }^{(18)}$. Soma-se a isso o fato de que as mulheres frequentam mais os centros de saúde do que os homens ${ }^{(19)}$. Dessa maneira, quanto mais velho for o contingente estudado, maior será o número de mulheres viúvas participantes nas pesquisas.

A relação gênero e capacidade funcional não foi estatisticamente significativa. Resultado semelhante foi apresentado por demais estudos da área ${ }^{(20-22)}$. Entretanto, alguns autores concluem que as mulheres apresentam duas vezes mais chances de desenvolver dependência do que os homens ${ }^{(11)}$. Apesar das mulheres apresentarem maior longevidade e maiores limitações ou perda da capacidade funcional, ainda não houve nenhum estudo que explicasse o motivo da perda de capacidade funcional mais acentuada no sexo feminino ${ }^{(23)}$.

A variável idade apresentou significância apenas quando relacionada com a MIF total $(\mathrm{p}=0,004)$. Entretanto, em outro estudo realizado com idosos, observa-se que tanto a MIF total, quanto a cognitiva e a motora demonstraram significância estatística ${ }^{(21)}$. Portanto, entende-se que a idade é um fator determinante na capacidade funcional entre idosos, isto porque, entre os 65 e 69 anos de idade, a chance do idoso desenvolver dependência é de aproximadamente 1,9 vezes, aumentando gradativamente até os 80 anos, quando a chance se torna 36 vezes maior ${ }^{(11)}$.

O grande número de idosos oriundos do meio rural $(63,1 \%)$ explica, em parte, o baixo nível de escolaridade dos idosos longevos. Segundo dados do Instituto Brasileiro de Geografia e Estatística, no Brasil, a média de anos estudados por um indivíduo com idade de 15 anos ou mais que reside em meio urbano é de 7,4, já um indivíduo do meio rural possui média de quatro anos de estudo ${ }^{(24)}$. Entende-se que os idosos da pesquisa, quando mais jovens, tiveram que optar pelo trabalho em detrimento ao estudo, para colaborar com a renda da família. Além disso, o acesso à escola era difícil, especificamente em relação ao tempo que demandavam as longas distâncias percorridas por meio de transporte animal.

Um estudo que analisou os dados socioeconômicos de 117 idosos longevos residentes no meio rural do Rio Grande do Sul observou que 58,8\% dos entrevistados eram analfabetos ou possuíam baixa escolaridade ${ }^{(25)}$. Outras pesquisas também apontam a baixa escolaridade de idosos longevos, os quais apresentaram, respectivamente, percentual de $60,1 \%$ e $75 \%$ de idosos analfabetos ou que não concluíram o ensino fundamental ${ }^{(4,16)}$. No presente estudo não houve relação significativa entre a MIF e a variável escolaridade.

A relação local de nascimento e capacidade funcional mostrou-se significativa para a variável MIF total $(p=0,029)$. Entende-se que o idoso oriundo do meio rural, com baixo nível socioeconômico possui maior chance de desenvolver incapacidades funcionais do que aqueles oriundos do meio urbano, com renda e acesso a informações. Entretanto, uma investigação, que avaliou a capacidade funcional de idosos longevos residentes em uma cidade com características interioranas, encontrou que 71,3\% dos entrevistados possuíam capacidade funcional avaliada como boa ou muito boa ${ }^{(6)}$. Segundo os autores, o fato dos idosos realizarem mais caminhadas, saírem à rua para conversar com os vizinhos, e serem mais ativos auxilia na sua independência e autonomia ${ }^{(6)}$. Nesse sentido, pode-se inferir que, quando os idosos longevos melhoram os níveis de participação, ou seja, tornam-se mais sociáveis, estarão fortalecendo a sua capacidade funcional.

A variável renda mostrou associação significativa à variável MIF total $(p=0,048)$. Uma boa condição econômica é um dado importante, pois permite ao longevo o acesso às informações, num amplo sentido, para um viver saudável e o acesso aos serviços de saúde de qualidade. A baixa condição econômica dos idosos longevos é um desafio nacional. Para superá-lo requer reformas políticas, que se alicercem em um olhar atento para o processo de envelhecimento de nossa sociedade, e para o idoso como contribuinte ativo.

A baixa renda dos idosos longevos foi encontrada em outros estudos realizados com essa população( ${ }^{(4,16-17)}$. Em idades mais avançadas, a possibilidade de obter um 
emprego é menor, resultando ao idoso receber apenas sua aposentadoria ou pensão como fonte de renda ${ }^{(26)}$.

Apesar da maioria dos idosos longevos residirem com familiares $(46,2 \%)$ os dados apontam alto percentual daqueles que residem sozinhos $(29,2 \%)$. A opção de viver sozinho pode refletir preferências pessoais e pode ser um estágio temporário no ciclo de vida do indivíduo ${ }^{(4)}$. A proporção de idosos mais velhos vivendo sozinhos é maior do que a observada em idosos mais jovens ${ }^{(4)}$. À medida que as pessoas envelhecem passam a ser mais seletivas e a ter hábitos de vida enraizados. Desse modo, apresentam dificuldades para alterar seus hábitos pessoais, mudança de residência e tornar-se sociável. Isso explica, em parte, a resistência em compartilhar residência com familiares e a preferência por morar sozinhos, muitas vezes, mesmo sem ter condições totais de independência e autonomia.

A variável com quem mora mostrou-se estatisticamente significativa para a MIF total $(\mathrm{p}=0,050)$. Morar sozinho pode ser um fator protetor para a independência, provavelmente em virtude desses idosos ainda estarem aptos física e economicamente ${ }^{(27)}$. Entretanto, é necessário atentar para as condições de moradia e hábitos de vida dos idosos, com a finalidade de evitar acidentes que possam resultar em dependências ${ }^{(21)}$.

Não possuir trabalho também foi uma variável significativa ao estudo $(\mathrm{p}=0,50)$ quando comparada a capacidade funcional dos longevos. Os idosos que ainda estão inseridos no mercado de trabalho e permanecem ativos na sociedade possuem maiores níveis de independência e autonomia. Porém, observa-se que o quantitativo de idosos longevos ainda inseridos no mercado de trabalho é baixo $(4,6 \%)$. Nesse contexto, existe a necessidade de implementação das políticas públicas de saúde que oportunizam o envelhecimento ativo, para permitir que os idosos continuem a trabalhar de acordo com suas capacidades e preferências, o que auxiliaria na prevenção de doenças crônicas e ou pelo menos a retardar as incapacidades.

\section{CONCLUSÕES}

Infere-se que a prevalência das características socioeconômicos de idosos longevos do gênero feminino, viúvas, baixa escolaridade e renda reafirma os resultados encontrados na maioria dos estudos com essa população específica. A feminização da velhice é um fenômeno mundial que necessita de atenção. As mulheres em idades mais avançadas, quando comparadas aos homens, possuem a saúde mais debilitada, com maior número de doenças e chances de desenvolver dependência e pior qualidade de vida no processo de envelhecimento.

Desse modo, a existência de estudos que avaliam as condições de saúde das idosas com mais de 80 anos auxiliariam no desenvolvimento de ações de cuidado, com vista ao aumento da qualidade de vida e promoção de uma velhice mais digna ao gênero feminino da população brasileira.

Além disso, identificar as variáveis que mostram associação significativa à capacidade funcional favorece as ações de enfermagem e os cuidados gerontológicos voltados para os idosos e suas famílias, as quais tem como objetivo uma assistência individualizada e específica à esta população. Determinar a capacidade funcional dos idosos longevos, bem como, a interferência de características sociais e demográficas nessa capacidade é elementar para a implementação de um plano de cuidados efetivo e eficaz para esses usuários da atenção básica de saúde.

Para a pesquisa em enfermagem, embora os resultados sejam locais, a contribuição está no despertar para a temática e necessidade de estudos na área, especificamente aqueles do tipo longitudinal, que visam o seguimento da amostra estudada.

\section{REFERÊNCIAS}

1. Caldas CP. Quarta idade: a nova fronteira da gerontologia. In: Papaléo Netto M, organizador. Tratado de gerontologia. $2^{\mathrm{a}}$ ed. São Paulo: Atheneu; 2007.

2. Nogueira SL. Capacidade funcional, nível de atividade física e condições de saúde de idosos longevos: um estudo epidemiológico [dissertação]. Viçosa (MG): Universidade Federal de Viçosa; 2008.

3. Inouye K, Pedrazzani ES, Pavarini SC. Octogenários e cuidadores: perfil sócio-demográfico e correlação da variável qualidade de vida. Texto ContextoEnferm. [Internet] 2008;17(2) [acesso em 18 fev 2013]. Disponível: http://www.scielo.br/pdf/tce/v17n2/18.pdf

4. Rosset I, Roriz-Cruz M, Santos JLF, Haas VJ, Fabrício-Wehbe SCC, Rodrigues RAP. Diferenciais socioeconômicos e de saúde entre duas comunidades de idosos longevos. Rev. Saúde Públ. [Internet] 2011;45(2) [acesso em 18 fev 2013]. Disponível: http://www.scielo. br/pdf/rsp/v45n2/1761.pdf

5. Freitas EV. Tratado de geriatria e gerontologia. $2^{\mathrm{a}}$ ed. Rio de Janeiro: Guanabara Koogan; 2006. 
6. Nogueira SL, Ribeiro RCL, Rosado LEFPL, Franceschini SCC, Ribeiro AQ, Pereira ET. Fatores determinantes da capacidade funcional em idosos longevos. Rev. Bras. Fisioter. [Internet]. 2010;14(4) [acesso em 18 fev 2013]. Disponível: http://www.scielo.br/pdf/rbfis/v14n4/aop019_10.pdf

7. Cordeiro RC, Dias RC, Dias JMD, Perracini M, Ramos LR. Concordância entre observadores de um protocolo de avaliação fisioterapêutica em idosas institucionalizadas. Rev Fisioter Univ São Paulo. [Internet]. 2002;9 [acesso em 18 fev 2013]. Disponível: http://rfp-ptr.com.br/download/2002/FPv9n2-2002.pdf

8. Nybo H, Petersen HC, Gaist D, Jeune B, Andersen K, Mcgue $\mathrm{M}$, et al. Predictors of mortality in 2,249 nonagenarians - the Danish 1905 - cohort survey. J Am Geriatr Soc. [Internet]. 2003;51(10) [acesso em 18 fev 2013]. Disponível: http://user. mpidr.de/jwv/pdf/Nybo-predictor.pdf

9. Femia EE, Zarit SH, Johansson B. The disablement process in very late life. A study of the oldest-old in Sweden. J Gerontol B Psychol. [Internet]. 2001; 56(1) [acesso em 18 fev 2013]. Disponível: http:// psychsocgerontology.oxfordjournals.org/content/56/1/ P12.full.pdf

10. Yi Z, Vaupel JW. Functional capacity and selfevaluation of health and life of oldest old in China. JSI. [Internet]. 2002;58(4) [acesso em 18 fev 2013]. Disponível:http://www.demogr.mpg.de/publications\% 5Cfiles\%5C1994_1122027333_1_JSocialIssue58-4.pdf

11. Rosa TEC. Benício MD, Latorre RDO; Ramos LR. Fatores determinantes da capacidade funcional entre idosos. Rev. Saúde Públ. [Internet]. 2003; 37(1) [acesso em 18 fev 2013]. Disponível: http://www.scielo.br/pdf/ tce/v17n2/18.pdf

12. Folstein MF, Folstein SE, Mchugh PR. "Mini-mental state". A practical method for grading the cognitive state of patients for the clinician. J Psychiatr Res. [Internet]. 1975;12(3) [acesso em 18 fev 2013]. Disponível: http://home.uchicago.edu/ /tmurrayl/ research/articles/printed $\% 20$ and $\% 20 \mathrm{read} / \mathrm{mini} \% 20$ mental $\% 20$ state_a $\% 20$ practical $\% 20$ method $\% 20$ for $\% 20$ grading $\% 20$ the $\% 20$ cognitive $\% 20$ state $\% 20$ of $\% 20$ patients $\% 20$ for $\% 20$ the $\% 20$ clinician.pdf

13. Bertolucci PH, Brucki SM, Campacci SR, Juliano Y. The mini-mental state examination in a general population: impact of educational status. Arq. NeuroPsiquiatr. [Internet]. 1994;52(1) [acesso em 18 fev 2013]. Disponível: http://www.scielo.br/scielo.php?script=sci pdf\&pid=S0004282X1994000100001\&lng=en\&nrm $=\overline{\mathrm{i}}$ so\&tlng $=\mathrm{pt}$
14. Riberto M, Miyazaki MH, Jorge Filho D, Sakamoto H, Battistella LR. Reprodutibilidade da versão brasileira da Medida de Independência Funcional. Acta Fisiátr. [Internet] 2001;8(1) [acesso em 18 fev 2013]. Disponível: www.innovarecursos.com.br/site/artigos/get/id/35

15. Riberto M, Miyazaki MH, Jucá SSH, Sakamoto H, Pinto PPN, Battistella LR. Validação da versão Brasileira da Medida de Independência Funcional. Acta Fisiátr. [Internet]. 2004;11(2) [acesso em 18 fev 2013]. Disponível: www.innovarecursos.com.br/site/artigos/ get/id/36

16. Inouye K, Pedrazzani ES. Nível de instrução, status econômico e avaliação de algumas dimensões da qualidade de vida de octagenários. Rev. LatinoAm. Enfermagem. [Internet] 2007 [acesso em $18 \mathrm{fev}$ 2013];15(n.esp). Disponível: http://www.scielo.br/pdf/ rlae/v15nspe/pt_04.pdf

17. Soares MBO, Tavares DMS, Dias FA, Diniz MA, Machado ARM. Características sociodemográficas, econômicas e de saúde de idosas octogenárias. Cienc. cuid saude. [Internet]. 2009;8(3) [acesso em $18 \mathrm{fev}$ 2013]. Disponível: http://periodicos.uem.br/ojs/index. php/CiencCuidSaude/article/viewFile/9046/5014

18. Instituto Brasileiro de Geografia e Estatística (IBGE). Síntese de Indicadores Sociais. Uma análise das condições de vida da população brasileira. Rio de Janeiro; 2009. [acesso em 21 de ago 2011]. Disponível: http://biblioteca.ibge.gov.br/visualizacao/monografias/ GEBIS $\% 20-\%$.

19. Lima LCV, Bueno CMLB. Envelhecimento e gênero: a vulnerabilidade de idosas no Brasil. Rev Saúde Pesquisa. [Internet] 2009;2(2). [acesso em $18 \mathrm{fev}$ 2013]. Disponível: http://www.cesumar.com.br/ pesquisa/periodicos/index.php/saudpesq/article/ viewFile/1173/792

20. Alvarenga MRM, Oliveira MAC, Faccenda O, Souza RA. Perfil social e funcional de idosos assistidos pela estratégia da saúde da família. Cogitare enferm. [Internet]. 2011;16(3) [acesso em 18 fev 2013]. Disponível: http://ojs.c3sl.ufpr.br/ojs2/index.php/ cogitare/article/viewfile/20944/16233

21. Amaral JG. Medida de independência funcional em idosos portadores de doença crônica [dissertação]. Garulhos (SP): Universidade de Guarulhos; 2010.

22. Monteiro RC, Faro ACM. Avaliação funcional de idoso vítima de fraturas na hospitalização e no domicílio. Rev Esc Enferm USP. [Internet]. 2010;44(3) [acesso em 18 
fev 2013]. Disponível: http://www.scielo.br/pdf/reeusp/ v44n3/24.pdf

23. Fiedler MM; Peres KG. Capacidade funcional e fatores associados em idosos do Sul do Brasil: um estudo de base populacional. Cad Saúde Pública. [Intenet] 2008;24(2) [acesso em 18 fev 2013]. Disponível: http:// www.scielo.br/pdf/csp/v24n2/19.pdf

24. Instituto Brasileiro de Geografia e Estatística (IBGE) [Internet]. Indicadores Sociodemográficos e de Saúde no Brasil [acesso em 30 out 2011]. Disponível: http:// www.ibge.gov.br.

25. Morais EP, Rodrigues RAP, Gerhardt TE. Os idosos mais velhos no meio rural: realidade de vida e saúde de uma população do interior gaúcho. Texto ContextoEnferm. [Internet] 2008;17(2) [acesso em 18 fev 2013]. Disponível: http://www.scielo.br/pdf/tce/v17n2/21.pdf

26. Telles SMBS. Idoso: família trabalho e previdência [tese]. Campinas (SP): Universidade Estadual de Campinas; 2003.

27. Lebrão ML. SABE - Saúde, bem-estar e envelhecimento - O projeto SABE no município de São Paulo: uma abordagem inicial. Brasília: Organização PanAmericana da Saúde; 2003. 